\title{
Differences of Learning Linguistic Skills Between ASD Children and Typical Developing Children
}

\author{
Zhuwei Shen ${ }^{1}$, Huiwen $\mathrm{Ma}^{2}$,*, Weiming $\mathrm{Chen}^{3}$, Mohan $\mathrm{Li}^{4}$ \\ ${ }^{1}$ School of science and technology, Wenzhou-Kean University, Wenzhou, Zhejiang 325060, China \\ ${ }^{2}$ Trinity High school, Camp Hill, PA, 17011, United States \\ ${ }^{3}$ Shanghai World Foreign Language Middle School, Shanghai, 200233, China \\ ${ }^{4}$ Donovan Catholic School Toms River, 08753, United States \\ *Corresponding author.Email:HMA.2022@THSROCKS.US
}

\begin{abstract}
About one in 68 children in China has autism, and this group should be given special attention. We couldn't help thinking the way they acquire language. Do they understand what typical developing kids understand? We assumed that autistic children are able to understand implicature and apply it. Testing is composed three parts totally and targeting two groups of kids (ASD and TD). We adapted Kissine and Cano-Chervel's (2015) experiment on context-based comprehension of indirect requests. We presented the experiment to the children in a more fun and relaxing way. In the first stage, children will be taught to assemble a toy, which is what they supposed to do in the later stage. Sooner, children will be requested in an indirect way, if they do understand it they will comply. We think that children with autism can understand between the lines if we get one pattern of data.
\end{abstract}

Keywords : implicature, autism spectrum disorder, children

\section{INTRODUCTION}

Autism is a kind of psychiatric illness. The main symptom of this disease is the lack of social development, communication skill and also along with repeating fixed behaviors (Landa, 2008)[1]. The fact that children have much more synapses than adults, which means they memorize things faster, made us target ASD children instead of ASD adults. Broadly speaking, we want to carry out a research on the differences of language development between ASD children and TD children. Specifically, do ASD children perform differently from children without ASD in understanding implicatures, which is regarded as an important linguistic skill? An implicature is what is suggested in an utterance though not expressed straightforwardly (Grice, 1975)[2]. An example of conversational implicature can be found in classroom. Student A: "Are you interested in Calculus?" and the student 2 answers: "UMMM, I mean I didn't jump for joy before class." It can be implied that the student 2 is not really interested in Cal through "didn't jump for joy". Therefore, this is answering the student 1 's question in an indirect fashion.

\section{BACKGROUND INFORMATION}

A large number of studies have focused on the impacts of autism on children's language development. Rapin and Dunn (1997) found that most children with autism are negatively affected or delayed in understanding and using language.[3] For example, the language of children with autism has two major defects. One is related to phonetics or language processing. The other one is that they may have obstacles when it comes to higher-level language processing. For example, they know "why" should be answered with "because", but they may answer this with an unrelated content. However, these defects are not permanent and can be solved through special educational means in special preschool. Thus, it is not a strong case to prove that children with autism cannot understand implicature compared to normal children.

In later research, Kissine and Cano-Chervel (2015) thought it can be concluded that children with autism understand indirect requests relying on certain contextual cues.[4] For example, "Can you close the window?" This sentence is not to ask you whether you have the ability to open this window, but to let you do this behavior, let you 
open the window. In this experiment, there are several shortcomings. In the comparison group, the normal children were too young and didn't have enough thinking ability, resulting in the normal children's scores lower than the ASD children. Generally speaking, participants with ASD choose fewer correct outcomes than the normal children group.

Additionally, Pastor-Cerezuela etc. (2018) did an experiment to evaluate the difference in the comprehension of GCI (Generalized Conversational Implicatures) between children with and without ASD.[5] This comprehension is specifically tested through 3 types of implicature: Q type means that the unmentioned content will not happen, I type means that there is no need to say anything can be assumed, the simple description is a typical feature. M type means speaking in an abnormal way. Sentences with these 3 types of implicature are given, and subjects are asked to choose from 3 interpretations regarding each of them. The entire experiment records the number of correct answers for each subject and the time they take to perform the test. The whole experiment divided the participants into three groups. The results showed that there were significant differences between the three groups, and the ASD group performed the worst. Compared to the Kissine and Cano-Chervel's(2015) experiment, Children with very low cognitive function are not included in this experimental sample.

With the continuous development of research in this area, the research topics have become more and more detailed. Beatriz López and Susan R. Leekam(2003) analyzed whether children with autism have the ability to process information in context compared to normal children.[6] Context refers to the background knowledge that is helpful for people to understand the meaning of a sentence or an article. Through visual (presentation through images), semantics, and homographs, this study conducted four experiments to prove whether children with autism have the ability to process information based on context. In this study, ASD children can recognize objects faster and more accurately in quite scenes than in noisy scenes. Whether or not visual information is provided, children with autism will consider context. In addition, through the detection of homographs, children with autism will be impaired when using sentence context in this task. This means that children with autism still have problems in integrating multiple information items to make choices. This experiment has provided us with a lot of help. For example, they innovated experimental methods. They transformed verbal tasks into visual tasks.

Based on the above, we hold the opinion that ASD children do not have trouble on understanding implicature which throw light on our topic.

\section{PROPOSED STUDY}

Do ASD kids understand what implicature is? Do they know how to use this language skill? Two alternatives come out after we searched related materials (the studies above).

A1 (alternative 1) would be the case that ASD kids can understand what people mean with the use of implicature. A possibility is that they behave similarly with normal kids.

A2 would be the case that ASD kids have trouble on understanding this pragmatic device. First, even if ASD kids can speak, they can't communicate. Their language are mostly self-talk, just like a parrot, keep repeating a single word or a sentence. Simply, they live in their own world.

Now, for our experiment, we use hidden cameras for the sake of experimental accuracy. Their excitement, fear, any mood swings can affect the experiment results. Besides, we choose the Olaf, a fictional character from Disney's Frozen franchise that is pretty closed to children daily life, as the object to be assembled. The test way, if they know that they were taking a test or an experiment, they will have pressure somehow.

\section{METHOD}

\subsection{PARTICIPATION}

We will set up two reference groups, one consisting of 12 children aged 5-7 years with typical development, the other consisting of 12 children aged 7-10 years with ASD. ASD kids in this age group are chosen because they develop language relatively slowly. For the size of participants, we use 12 because this is an average number of ranges from all studies we searched online. The number of sample is neither too small to ensure its broad applicability nor too large to do united measuring. All children are native English speaker and had received a formal diagnosis of autism in conformity to the criteria of DSM-IV by a team of professional neuropsychologists, pediatricians and speech therapists specialized in ASD diagnosis.[4] All parents of participants have filled in informed consent forms.

\subsection{PRE-TEST}

In a private room, children would be separated and explained individually by Experimenter that how to assemble Olaf, EXP will also use some generated utterances (e.g. "Could you please try to put the nose on him?") to encourage or to prompt them to search and attach parts in the process. So, the purpose of the pre-test is, first, making sure all of the kids understand how to assemble the object, second, emphasizing the directive interpretation from the Experimenter.[4] 


\subsection{PROCEDURE}

The whole experiment will be taken in a school for a whole day. Each kid will be invited into a quiet classroom, one at a time, with three hidden cameras, a chair and a low table. Every single word they say, behaviors of them will be recorded. Children may feel uncomfortable in the front of the camera, so we use hidden one for sake of it.

Kids are given a half-completed Olaf. Experimenter will say the target-sentence:" Oh My God! He has no nose!" as a hint during the individual conversation. Obviously, the target-sentence is a request here: to put the nose on Olaf. To ensure the validity of our results, we will conduct this experiment three times on each kids individually, but with different target-sentence, for trial 2 the EXP will say: "Don't you feel like the little Olaf is lack of a hat?" the target-sentence for trial 3 is similar with the one in trial 1 , but we use tail instead of nose. The goal of it is to determine whether the participants are able to get the indirect meaning of the target-sentence and perform correctly.[4]

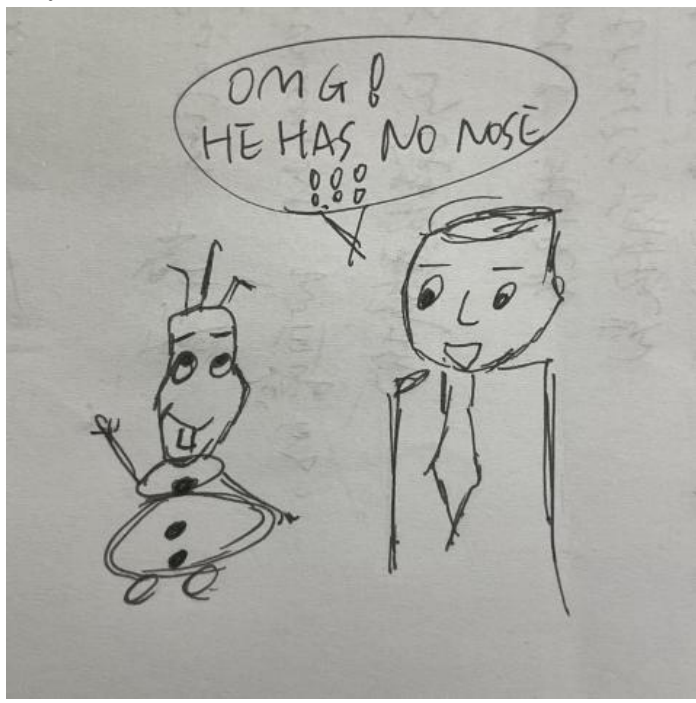

Figure 1 : OMG. He has no nose

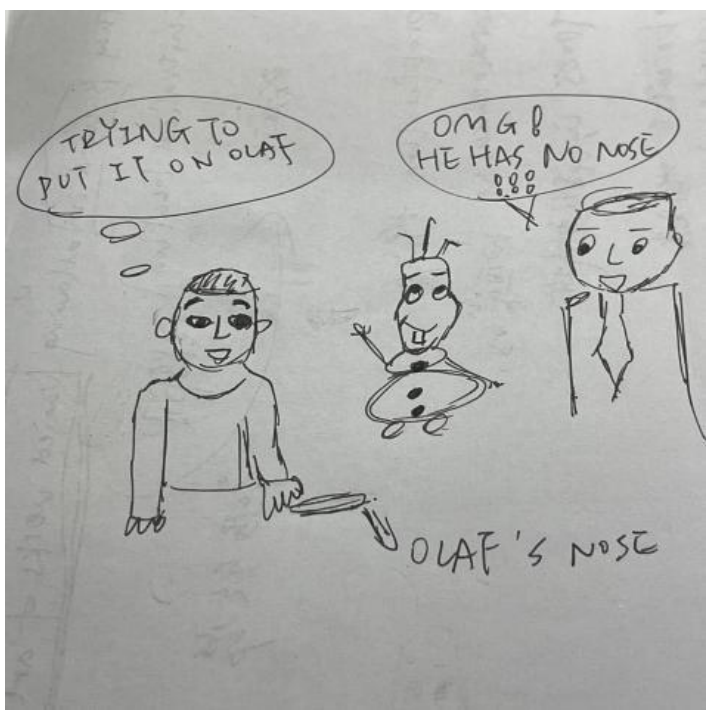

Figure 2: put the nose on Olaf

\subsection{SCORING :}

EXP will score all 24 kids. The maximum score of 2 is assigned when the child complies with the indirect request; that is, they do really put the nose/hat/tail on the Olaf. The intermediate score of 1 is assigned when the child has a clear tend or a gesture towards reaching the nose/hat/tail but did not take action in the end. And no compliance is marked 0 .
All kids will be told to keep the conversation as a secret.

\section{RESULTS AND DISCUSSION}

The total possible score of the experiment is six. If the participants get zero, which means they can't understand the implication at all. If they get one to three points, that means they can make responses, but the 
responses may not be fully correct. This explains that they can't clearly understand the meaning behind the line. And if they get 3-5 points, they can make a certain correct response, but because there may be some delays or negative effects of autism, such as inability to concentrate, they may not be able to get full marks. If they get 6 points, which means that they can understand implication well. And we suppose that autistic kids can understand the implicature.
Also, we will do the same experiments on the normal children group. And the normal children may not get full marks because they may be affected by the age.

Finally, we can get a similar chart as shown in Figure 1 , the horizontal length of the data represents the level of their scores. The comparison of the length of lines in different colors shows the difference between groups, it represents the sum of the score of each group in each experiment.

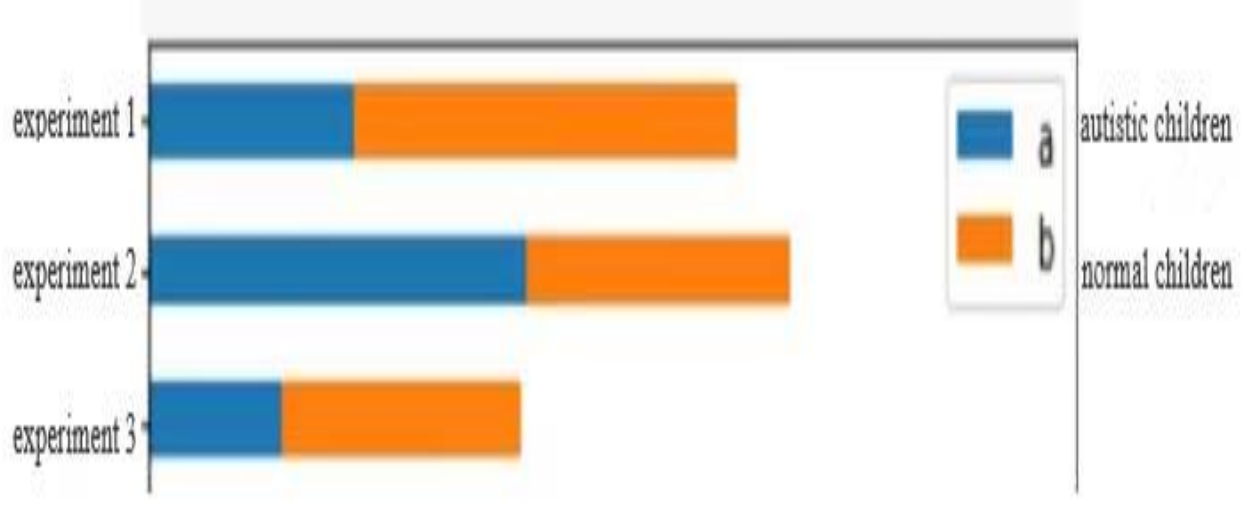

Figure 3 The comparison of score normal children and autistic children get in 3 experiments

\section{CONCLUSION AND FUTURE DIRECTIONS}

This experiment can explain that children with autism can understand the meaning of words if we get one pattern of data. However, there are still some limitations in our experiment. First, we did not compare different age groups, and did not longitudinally consider the impact of age factors on the language development of ASD children. In addition, we set up only one set of data for ASD groups and TD groups, each with only 12 children. Therefore, data errors of a single sample will have a significant impact on the overall results. In the future, more groups of data can be added for comparison, for example, the number of samples can range at 50 per group.

In this experiment, we only studied one specific kind of language development. Therefore, not only in terms of implicature, but we can also study whether repetition, adjectives, etc. influence ASD children. In addition, we can also study whether children with autism will be affected when they learn bilingualism. Not only that, but we can also study the difference in language between ASD children and normal children throughout their lives.

\section{REFERENCES}

[1] Landa, R. J. (2008). Diagnosis of autism spectrum disorders in the first 3 years of life. Nature Clinical Practice Neurology volume , 138-147.
[2] Grice, H. P. (1975). Logic and conversation. In Speech acts (pp. 41-58). Brill.

[3] Rapin, I., \& Dunn, M. (1997, June). Language disorders in children with autism. In Seminars in Pediatric Neurology (Vol. 4, No. 2, pp. 86-92). WB Saunders.

[4] Kissine, M., Cano-Chervel, J., Carlier, S., De Brabanter, P., Ducenne, L., Pairon, M. C., ... \& Leybaert, J. (2015). Children with autism understand indirect speech acts: Evidence from a semi-structured act-out task. PLoS One, 10(11), e0142191.

[5] Pastor-Cerezuela, G., Tordera Yllescas, J. C., González-Sala, F., Montagut-Asunción, M., \& Fernández-Andrés, M. I. (2018). Comprehension of generalized conversational implicatures by children with and without autism spectrum disorder. Frontiers in psychology, 9, 272.

[6] Lopez, B., \& Leekam, S. R. (2003). Do children with autism fail to process Information in context?. Journal of child psychology and psychiatry, 44(2), 285-300. 